Homology, Homotopy and Applications, vol.7(2), 2005, pp.139-160

\title{
ALGEBRAIC MODELS FOR HOMOTOPY TYPES
}

\author{
JULIO RUBIO AND FRANCIS SERGERAERT
}

\author{
(communicated by James Stasheff)
}

\begin{abstract}
The classical problem of algebraic models for homotopy types is precisely stated here in terms of our ability to compute with the models. Two different natural statements for this problem are produced, the simplest one being entirely solved by

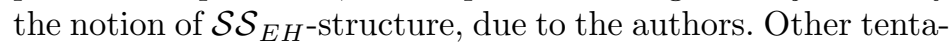
tive solutions, Postnikov towers and $E_{\infty}$-chain complexes, are considered and compared with the $\mathcal{S S}_{E H \text {-structures. In partic- }}$ ular, an imprecision in the usual definition of the $k$ - "invariants" is explained, which implies we seem far from a solution for the ideal statement of our problem. On the positive side, the problem stated below in the framed quotation is solved.

As yet we are ignorant
of an effective method of computing
the cohomology of a Postnikov complex
from $\pi_{n}$ and $k^{n+1}[\mathbf{1 1}]$.
\end{abstract}

\section{Introduction ${ }^{1,2,3}$.}

Obtaining "algebraic" models for $\mathbb{Z}$-homotopy types is a major problem in algebraic topology. We explain in this article the importance of models which are computable (or effective, constructive, ....).

The problem of the title can then be precisely stated in two different ways, the hard problem (Problem 5 in Section 2) and the soft problem (Problem 8 in Section 3). The notion of simplicial set with effective homology $\left(\mathcal{S S}_{E H}\right)$, due to the authors, is a complete solution for the soft problem, very simple from a theoretical point of view, once the possibilities of functional programming are understood. This solution has led to an interesting concrete computer work, the Kenzo program,

Received October 15, 2003, revised February 12, 2004; published on April 22, 2005.

2000 Mathematics Subject Classification: 55P15, 55S45, 18-04, 18G40, 18G55, 55-04, 55P35, 55R20, 55T10, 55T20.

Key words and phrases: Classification, homotopy type, Postnikov system, k-invariant, computable model, algebraic model, computability.

(C) 2005, Julio Rubio and Francis Sergeraert. Permission to copy for private use granted.

${ }^{1}$ This text is a slightly expanded version of a talk given by the authors at the RSME-AMS meeting at Sevilla in June 2003, which explains its nature a little expository. The talk itself is available as a dvi-file at www-fourier.ujf-grenoble.fr/ ${ }^{\sim}$ sergerar/Talks.

${ }^{2}$ The authors would like to express their thanks to Jim Stasheff for his careful reading of the first submitted version, and for his numerous and helpful suggestions.

${ }^{3}$ First author's work is partially supported by MCyT, project TIC2002-01626 and by CAR ACPI2002/06. 
demonstrated a little in the article to give to the reader some experimental evidence that the stated results are correct.

Other solutions for the soft problem could be based on operadic techniques, and they are now intensively looked for. The key point is the notion of $E_{\infty}$-operad; a broad outline of the main results so obtained is given and compared with the $\mathcal{S S}_{E H}$ solution. The current result is that the $\mathcal{S S}_{E H}$ solution is, for the soft problem, simple and already implemented. The operadic structures are of course interesting, give many useful informations, but can be regarded as by-products of $\mathcal{S S}_{E H}$-structures; furthermore it is not clear how they could produce autonomous computable objects. A good point of view for future work is probably a mixture of $\mathcal{S} \mathcal{S}_{E H}$ 's and operadic techniques, the last ones to be considered as good tools to better understand and also to improve the computability results so easily obtained through $\mathcal{S S}_{E H}$ 's.

The hard problem is thus reduced to the problem of equivalence between sets of $k$-invariants, a problem which, as far as we know, seems open: we explain why the so-called $k$-invariants are not actual invariants so that finally the standard Postnikov theory does not solve the hard problem.

\section{An improved statement of the problem.}

The construction of algebraic models for homotopy types is a "classical" problem in Algebraic Topology. Most topologists should agree with the following statement of our problem.

Problem 1. - Let $\mathcal{H}$ be the homotopy category. How to design an algebraic category $\mathcal{A}$ and a functor $F: \mathcal{H} \rightarrow \mathcal{A}$ which is an equivalence of categories?

Instead of working in the category $\mathcal{H}$, reputed to be a difficult category, you might work in the category $\mathcal{A}$, an algebraic category, hence probably a more convenient workspace. The image $F(X)$ of some homotopy type $X$ would be an algebraic object, for example a chain complex provided with a sufficiently rich structure to entirely define a homotopy type. Problem 1 leads to an auxiliary problem.

Problem 2. - What is the definition of an algebraic category?

The following example should give a better understanding of this problem. Many topologists consider a simplicial set not as an algebraic object. A simplicial set $S$ is a sequence of sets $\left(S_{n}\right)$ combined with some sets of operators between these sets, appropriate composites of these operators having to satisfy a few simple relations. Most topologists consider a chain complex $C_{*}$ provided with a module structure with respect to some (... algebraic!) operad $\mathcal{O}$ is an algebraic object. Such a chain complex is a sequence of chain groups $\left(C_{n}\right)$ combined with some sets of operators between these chain groups and their tensor products, appropriate composites of these operators having to satisfy a large set of sophisticated relations. Where is the basic difference? This appreciation - an $\mathcal{O}$-module is an algebraic object and a simplicial set is not - is arbitrary. Furthermore a simplicial structure is simpler than an $\mathcal{O}$-module structure, so that a beginner in the subject would probably guess the first structure type is "more" algebraic than the second one. Must we recall we are working in mathematics, not in philosophy? 
Terminology 3. - In our current mathematical environment, the border between algebraic objects and non-algebraic objects cannot be mathematically defined.

Let us continue our comparison between simplicial sets and chain complexes, which will eventually lead to the right point of view. The simplest example of an interesting result produced by Algebraic Topology is the Brouwer Fixed Point theorem, a direct consequence of the following.

Theorem 4. - Let $i_{n}: S^{n-1} \rightarrow D^{n}$ be the canonical inclusion of the $(n-1)$-sphere into the $n$-ball. There does not exist a continuous map $\rho_{n}: D^{n} \rightarrow S^{n-1}$ such that the composite $\rho_{n} \circ i_{n}$ is the identity map of $S^{n-1}$.

In fact, if you apply the $H_{n-1}$-functor to the data, the statement is transformed into: let $i: \mathbb{Z} \rightarrow 0$ be the null morphism; there does not exist a morphism $\rho: 0 \rightarrow \mathbb{Z}$ such that the composite $\rho \circ i$ is the identity morphism of $\mathbb{Z}$.

Most topologists think this process produces the result because the transformed problem has an algebraic nature, but this is erroneous. The algebraic qualifier is secondary; a better qualifier in fact is computable. The transformed problem is a particular case of the following: let $m, n$ and $p$ be three non-negative integers, and $f: \mathbb{Z}^{m} \rightarrow \mathbb{Z}^{n}$ and $F: \mathbb{Z}^{m} \rightarrow \mathbb{Z}^{p}$ be two $\mathbb{Z}$-linear morphisms; does there exist a morphism $g: \mathbb{Z}^{n} \rightarrow \mathbb{Z}^{p}$ satisfying $g \circ f=F$ ? It is common to think of this problem as an algebraic one, but in fact the important point for us is that there exists an algorithm giving the solution: a Smith reduction of the $\mathbb{Z}$-matrices representing $f$ and $F$ quickly gives the solution; in the case of the Brouwer problem, the Smith reduction is already done.

The previous considerations about simplicial sets gives us another idea. Because a simplicial set is in fact as "algebraic" as a homology group or a chain complex, why not work directly with simplicial models for $S^{n-1}$ and $D^{n}$ ? It is easy to give simplicial models with two (resp. three) non-degenerate simplices for $S^{n-1}$ (resp. $D^{n}$ ), models that are undoubtedly "algebraic". But these models have an essential failing: they do not satisfy the Kan extension condition, so that they are not appropriate for working in the homotopy category $\mathcal{H}$. In general the Kan simplicial models are highly infinite and cannot be directly used for computations: any tentative solution using in an essential way the Kan simplicial sets raises hard computability problems. We will see later that our solution for "algebraic" models for homotopy types is a simple but subtle combination of simplicial sets most often not of finite type with chain complexes of finite type.

There is a common fundamental confusion between the algebraic and computable qualifiers, still present in the ordinary understanding of the very nature of Algebraic Topology.

Let us look again at the statement of Problem 1. In fact, we are looking for a target category where automatic computations (pleonasm) can be undertaken. We thus obtain a new statement for our problem.

Problem 5. (Hard Problem) - Let $\mathcal{H}$ be the homotopy category. How to design a computable category $\mathcal{C}$ and a functor $F: \mathcal{H} \rightarrow \mathcal{C}$ which is an equivalence of categories? 
With the satellite problem:

Problem 6. - What is the definition of a computable category?

We do not want to consider the details of the last subject, an interesting subject, out of the scope of the present paper. Fundamentally different answers are possible, mainly from the following point of view: do you intend to apply the "computable" qualifier to the elements of an object in the category or to the objects themselves, or both? To our knowledge, the relevant corresponding theory is not yet settled ${ }^{4}$. The few examples given in this paper could be a guideline toward the most natural solutions of this question.

In this paper, a computable category is a category having properties roughly similar to those that are exhibited for our $\mathcal{S S}_{E H}$ category, and this approximate "definition" is here sufficient.

\section{Three tentative solutions.}

The current state of Algebraic Topology gives three main possibilities:

1. The Postnikov category;

2. The authors' solution: the category $\mathcal{S S}_{E H}$;

3. The operadic solutions.

In short, the first possibility is currently inadequate in the standard framework, because of an essential lack of computability, see the framed title inscription, and also because of the underlying classification problem which does not yet seem solved. The $\mathcal{S S}_{E H}$ category solves a subproblem, the "soft" problem stated a little later, and furthermore makes the Postnikov category computable; a consequence is the fact that the Postnikov category, when modelled as a satellite category of the $\mathcal{S S}_{E H}$ category, solves the same subproblem. It can be reasonably conjectured that the third idea, using operadic techniques, should in finite time solve the same subproblem, but we are still far from it, and this seems the challenge \#1 for the operad developers: how to organise the $E_{\infty}$-chain complexes as an autonomous computable category? The impressive concrete results obtained by V. Smirnov [25], at least when working with coefficients in a field $\mathbb{F}_{p}$, indicate that the vast theoretical study about $E_{\infty}$-chain complexes undertaken by this author could be a good guideline.

The gap about the classification problem remains present for the three techniques.

Once the theoretical and concrete possibilities of functional programming are understood, the $\mathcal{S S}_{E H}$ category is not too complicated, so that it has been possible to write down a computer program implementing the $\mathcal{S S}_{E H}$ category and to use it, see $[8]$ and Sections 5 and 7 of the present paper.

${ }^{4}$ For example, the interesting reference [19] is not sufficient for Problem 6; no tool is provided there for the equality problem between objects of a category. 


\subsection{The Postnikov category.}

Restriction 7. - Unless otherwise stated, all our topological spaces are connected and simply connected.

An object of the Postnikov category is a pair of sequences $\left(\left(\pi_{n}\right)_{n \geqslant 2},\left(k_{n}\right)_{n \geqslant 3}\right)$, made of homotopy groups and " $k$-invariants" defining a Postnikov tower $\left(X_{n}\right)_{n \geqslant 2}$. The first stage of the tower $X_{2}$ is $K\left(\pi_{2}, 2\right)$, the first $k$-invariant $k_{3} \in H^{4}\left(X_{2}, \pi_{3}\right)$ defines a fibration $X_{3} \rightarrow X_{2}$ the fiber being $K\left(\pi_{3}, 3\right)$, and so on. It is not hard to define the valid morphisms between two towers, and so we have defined the Postnikov category $\mathcal{P}$. We know it is not a common opinion, but this category is "algebraic".

The so-called $k$-invariants are not invariants, for the following reason: different $k$-invariants frequently give the same homotopy type. Identifying the corresponding equivalence classes is a problem which, to our knowledge, is yet without any general solution. Let us look at this simple example: what about the Postnikov towers with only $\pi_{2}=\mathbb{Z}^{p}, \pi_{5}=\mathbb{Z}$ and the other $\pi_{n}$ 's are null. The only relevant $k$-invariant is $k_{5} \in H^{6}\left(K\left(\pi_{2}, 2\right), \pi_{5}\right)=\operatorname{Cub}\left(\mathbb{Z}^{p}, \mathbb{Z}\right)$, the $\mathbb{Z}$-module of the cubical forms over $\mathbb{Z}^{p} ;$ making these cubical forms actual invariants amounts to being able to construct and describe in a computational way the quotient set $\operatorname{Cub}\left(\mathbb{Z}^{p}, \mathbb{Z}\right) /$ (linear equivalence). We have questioned several arithmeticians and they did not know whether appropriate references would allow a $k$-invariant user to solve this problem: the classification problem does not seem to be solved by the " $k$-invariants" and our example is one of the simplest ones ${ }^{5}$.

Let us quote certainly one of the best specialists in homotopy theory. Hans Baues says in $[\mathbf{4}$, p. 33]:

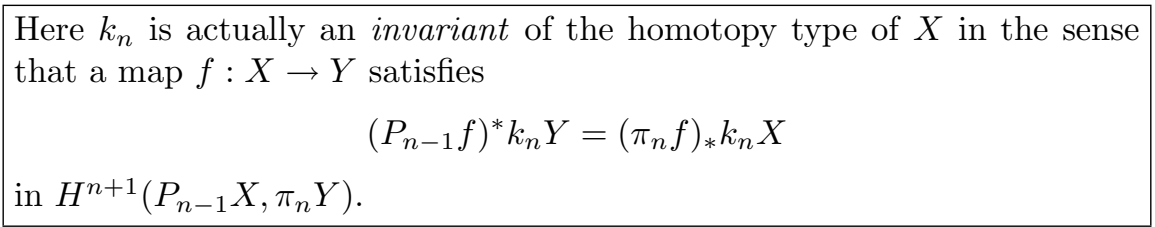

This is not strictly speaking the usual meaning of invariant; the cohomology class $k_{n}$ would be an actual invariant of the homotopy type if a homotopy equivalence $f: X \simeq Y$ implies $k_{n} X=k_{n} Y$; in fact the framed equal sign does not make sense: the underlying cohomology groups are not the same, they are only, in the relevant cases, isomorphic and two invariants should be considered as "equal" as soon as they are in turn "isomorphic" in an obvious sense. Baues' relation only shows the " $k$ invariant" depends functorially on the data, but it is not an invariant; the definition would be acceptable if the isomorphism problem between the various possible $k$ invariants in the same homotopy class had a (computable) solution, but the simple example given before shows such a solution does not seem currently known. We

${ }^{5}$ We would like to thank Daniel Lazard for his study (private communication) which opens several interesting research directions around this subject. 
will examine again this question in a more explicit way in Section 8, where another classical reference about $k$-invariants $[\mathbf{1 6}]$ is also studied.

This is probably the reason why Hans Baues uses entirely different techniques to obtain certainly the most interesting concrete results so far reached in the general classification problem; see [4, Section 11] and Baues' references in the same paper.

Let us consider the following subproblem of the hard one:

Problem 8. (Soft Problem) - How to design a computable category $\mathcal{C}$ and a functor $F: \mathcal{C} \rightarrow \mathcal{H}$ such that any recursive homotopy type is in the image of $F$ ?

In fact the hard problem as it is stated in Problem 5 cannot have a solution: the standard homotopy category $\mathcal{H}$ is much too rich to make it equivalent to a computable category. This is a situation analogous to which is well known for example for the real numbers. A computable real number is usually called a recursive real number and the set of the recursive real numbers is countable, much smaller than the set of "ordinary" real numbers, see [27]. In the same way:

Definition 9. - A recursive homotopy type is defined by a recursive Postnikov tower $\left(\left(\pi_{n}\right)_{n \geqslant 2},\left(k_{n}\right)_{n \geqslant 3}\right)$ : the data of this tower are defined by an algorithm $n \mapsto\left(\pi_{n}, k_{n}\right)$. In other words the recursive homotopy category is the image of the canonical functor $\mathcal{P}_{r} \rightarrow \mathcal{H}$ if $\mathcal{P}_{r}$ is the category of the recursive Postnikov towers.

In fact, in the standard context, this definition poses a problem: the required algorithm must be able to compute the $H^{n+1}\left(X_{n-1}, \pi_{n}\right)$ to allow it to "choose" the next $k_{n}$, and classical Algebraic Topology does not solve this question [11]. To our knowledge, there are currently only two solutions for this problem, independantly and simultaneously found by Rolf Schön $[\mathbf{2 0}]$ and the present authors $[\mathbf{2 1}, \mathbf{1 8}$, 23]. We will see later that our $\mathcal{S S}_{E H}$ category allows us in particular to compute the cohomology groups $H^{n+1}\left(X_{n-1}, \pi_{n}\right)$ when the previous data are available, so recursively defining where the $k_{n}$ is to be chosen. In this way our category $\mathcal{S S}_{E H}$ makes applicable Definition 9 and, then only, the Postnikov category becomes an obvious solution for the soft problem. In fact we will also see the $\mathcal{S S}_{E H}$ category directly gives a solution for the soft problem.

It should be clear now that in the statement of the hard problem, the category $\mathcal{H}$ must obviously be replaced by the category of the recursive homotopy types. Up to a finite dimension, this amounts only to requiring that the homotopy groups $\pi_{n}$ are of finite type, but if the situation is considered without any dimension limit, the requirement is much stronger.

Restriction 10. - From now on, all our categories are implicitely limited to recursive objects and recursive morphisms.

\subsection{The operadic solutions.}

Many interesting works have been and are currently undertaken to reach operadic solutions for the hard and for the soft problem. Probably the most advanced one is due to Michael Mandell [14], usually considered as a "terminal" solution. See also $[\mathbf{1 3}]$ for a $p$-adic environment. Of course we do not intend to reduce the interest of this essential work, but in Mandell's article the computability question is 
not considered, and the proposed solution invokes numerous layers of sophisticated techniques, so that the computational gap is not insignificant. In fact the interesting next problem raised by Mandell's paper is the following: is it possible to "naturally" extend Mandell's results to obtain the corresponding effective statements, or, on the contrary, is it necessary to add something which is essentially new and/or different? A solution is probably reachable in characteristic $p$, but if it was possible to obtain the same result with respect to the ground ring $\mathbb{Z}$, then crucial computability problems in arithmetic would be solved, problems which cannot be directly reduced to $\mathbb{Z}_{p}$-problems, see the discussion in Section 8.1.

The operadic techniques raise other essential difficulties: it seems extremely difficult to make computable the relevant categories. The challenge is the following: the ordinary constructions of algebraic topology - loop spaces, classifying spaces, fibrations for example - should have a translation in the chosen category. The now standard methods of closed model categories give many possibilities, give frequently elegant theoretical solutions for these translations, but the computational satellite problems are seldom studied. Let us note for example the difficulties met by the topologists when they iterate the Cobar construction.

However, as already observed, an entirely combinatorial translation of Smirnov's or Mandell's operadic techniques could be the right direction. Another research

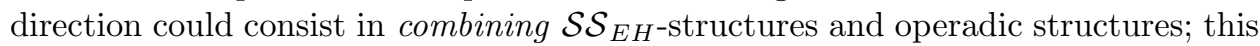
question will be examined in Section 9 .

\section{The category $\mathcal{S S}_{E H}$.}

Restriction 11. - All the chain complexes considered from now on are implicitly assumed to be free $\mathbb{Z}$-complexes, not necessarily of finite type.

The notion of reduction ${ }^{6}$ is well known.

Definition 12. - A reduction $\rho: C_{*} \Longrightarrow D_{*}$ between two chain complexes $C_{*}$ and $D_{*}$ is a triple $\rho=(f, g, h)$ where:

1. The first component $f$ is a chain complex morphism $f: C_{*} \rightarrow D_{*}$;

2. The second component $g$ is a chain complex morphism $g: D_{*} \rightarrow C_{*}$;

3. The third component $h$ is a homotopy operator (degree $=+1) h: C_{*} \rightarrow C_{*}$;

4. These components satisfy the relations:

(a) $f \circ h=0$;

(b) $h \circ g=0$;

(c) $h \circ h=0$;

(d) $\operatorname{id}_{D_{*}}=f \circ g$

(e) $\operatorname{id}_{C_{*}}=g \circ f+d_{C_{*}} \circ h+h \circ d_{C_{*}}$.

These relations express in an effective way how the "big" chain complex $C_{*}$ is the direct sum of the "small" one $D_{*}$ and an acyclic one, namely the kernel of $f$.

${ }^{6}$ Often called contraction or SDR data or ... 
Definition 13. - A strong chain equivalence (or simply an equivalence):

$$
\varepsilon: C_{*} D_{*}
$$

is a pair of reductions $\varepsilon=\left(\rho_{\ell}, \rho_{r}\right)$ where:

$$
C_{*} \stackrel{\rho_{\ell}}{\stackrel{\rho_{r}}{\rightleftharpoons}} \widehat{C}_{*}
$$

with $\widehat{C}_{*}$ some intermediate chain complex.

Definition 14. - A simplicial set with effective homology is a 4-tuple

$$
X_{E H}=\left(X, C_{*} X, E C_{*}^{X}, \varepsilon^{X}\right)
$$

where:

1. The first component $X$ is a locally effective simplicial set;

2. The second component $C_{*} X$ is the locally effective chain complex canonically associated to $X$;

3. The third component $E C_{*}^{X}$ is an effective chain complex;

4. The last component $\varepsilon^{X}$ is a strong chain equivalence $\varepsilon^{X}: C_{*} X \ldots E C_{*}^{X}$.

An effective chain complex is an ordinary object, no surprise; it is an algorithm $n \mapsto\left(C_{n}, d_{n}\right)$ where, for every integer $n$, the corresponding chain group $C_{n}$ is a free $\mathbb{Z}$-module of finite type, and $d_{n}$ is a $\mathbb{Z}$-matrix describing the boundary operator $d_{n}$ : $C_{n} \rightarrow C_{n-1}$. Elementary algorithms then allow us to compute the homology groups of such a complex. The third component $E C_{*}^{X}$ of a simplicial set with effective homology is of this sort.

A locally effective chain complex is quite different. It is an algorithm:

$$
n \mapsto\left(\chi_{n}, d_{n}\right)
$$

to be interpreted as follows.

1. The first component $\chi_{n}$ of a result is also an algorithm $\chi_{n}: \mathcal{U} \rightarrow\{\top, \perp\}$ where $\mathcal{U}$ (universe) is the set of all the machine objects, so that for every machine object $\omega$, the algorithm $\chi_{n}$ returns $\chi_{n}(\omega) \in\{\top, \perp\}$, that is, true or false, true if and only if $\omega$ is a generator of the $n$-th chain group of the underlying chain complex.

2. The second component $d_{n}$ of a result is again an algorithm: if $\chi_{n}(\omega)=T$, then $d_{n}(\omega)$ is defined and is the boundary of the generator $\omega$, therefore a finite $\mathbb{Z}$-combination of generators of degree $n-1$.

The set $\mathcal{U}$, for any reasonable machine model, is countably infinite, so that a locally effective chain complex in general is not of finite type. The adverb locally has the following meaning: if someone produces some (every!) generator $\omega$ of degree $n$, then the $d_{n}$-component is able to compute the boundary $d_{n}(\omega)$. The terminology generator-wise effective chain complex would be more precise but a little unwieldy.

A non-interesting but typical example of a locally effective chain complex would be produced by $\chi_{n}(\omega)=\top$ if and only if $\omega \in \mathbb{N}$, independently of $n$, and $d_{n}(\omega)=0$ for every $n \in \mathbb{Z}$ and $\omega \in \mathbb{N}$. In other words the underlying chain complex would be the periodic one $C_{n}=\mathbb{Z}^{(\mathbb{N})}$ with a null boundary. 
Standard logic shows in general the homology groups of a locally effective chain complex are not computable; this is an avatar of the Gödel-Turing-Church-Post theorems about incompleteness. More generally, global information in general cannot be deduced from a locally effective object. The second component $C_{*} X$ of a simplicial set with effective homology is of this sort.

A locally effective simplicial set is defined in the same way; the simplices are defined through characteristic algorithms $\chi_{n}$, and instead of computing boundaries, a set of appropriate operators computes faces and degeneracies.

The second component $C_{*} X$ of a simplicial set with effective homology is redundant: a simple algorithm can construct it from the locally effective simplicial set $X$; and strictly speaking, we could forget it in the presentation. But the key points in an object with effective homology are:

1. The main components are two $\mathbb{Z}$-free chain complexes $C_{*} X$ and $E C_{*}^{X}$, the first one being a direct consequence of the underlying object $X$, the second one describing the homology of this object, reachable through an elementary algorithm;

2. The component $C_{*} X$ is locally effective allowing it to be of non-finite type, with the drawback that in general its homology is not computable;

3. The component $E C_{*}^{X}$ is effective, therefore of finite type with computable homology;

4. The equivalence $\varepsilon^{X}$ is the key connection between the locally effective object $C_{*} X$ and the effective one $E C_{*}^{X}$.

and it is hoped the nature of this organization is better explained in the notation $\left(X, C_{*} X, E C_{*}^{X}, \varepsilon^{X}\right)$.

This notation is in fact a little misleading. A subtle difficulty is here which needs a constructivist viewpoint instead of the standard one - Zermelo-Fraenkel - to be understood. The locally effective subobject $X$ which is the first component of an object with effective homology does not effectively determine the "mathematical" underlying object $X$, because of the standard incompleteness theorems. Only very partial - "local" - information is reachable through such an object; if free colors were available in the text-processing system used when preparing this text, a very pale color should have been chosen for this symbol $X$, to clearly recall this sub-object is not $X$, but a new kind of object rarely considered in standard mathematics, an object of the third type [23].

Theorem 15. - The category $\mathcal{S S}_{E H}$ is a solution for the soft problem.

It is not possible in the framework of this paper to give a proof of Theorem 15, we will give only a demonstration. We apologize for the poor joke: "demonstration" has two different meanings in our context, it can be a mathematical proof, and it can be also a machine (computer) demonstration. It is expected in this case a machine demonstration should give the reader a strong conviction that the Kenzo program contains a proof of Theorem 15. This is the aim of Sections 5 and 7. 


\section{A small machine demonstration.}

This section uses a small machine demonstration to explain how, thanks to the powerful computer language Common Lisp, the Kenzo program [8] makes the objects and morphisms of the $\mathcal{S S}_{E H}$ category concretely available to the topologist.

Let us consider the following space:

$$
X=\Omega\left(\Omega\left(P^{\infty}(\mathbb{R}) / P^{3}(\mathbb{R})\right) \cup_{4} D^{4}\right) \cup_{2} D^{3}
$$

The infinite real projective space stunted to begin at dimension $4, P^{\infty}(\mathbb{R}) / P^{3}(\mathbb{R})$, is firstly considered; its loop space is constructed and the homotopy of this loop space begins with $\pi_{3}=\mathbb{Z}$; so that attaching a 4 -cell by a map $\partial D^{4} \rightarrow S^{3}$ of degree 4 makes sense and this is done. The loop space functor is again applied to the last space and finally a 3-cell is attached by a map of degree 2. This artificial space $X$ is chosen because it is not too complicated, yet it accumulates the main known obstacles to the theoretical and concrete computation of homology groups in small dimensions.

The space $X$ is an object of the category $\mathcal{S S}_{E H}$, so that the Kenzo program can construct it as such an object. The standard simplicial model of $P^{\infty}(\mathbb{R}) / P^{3}(\mathbb{R})$ has exactly one simplex in dimensions $0,4,5, \ldots$ : it is a simplicial set of finite type in any dimension; so that installing this space as an object of $\mathcal{S} \mathcal{S}_{E H}$ is straightforward. Kenzo knows this fact, and the predefined $r$-proj-space function implements it.

Now, using Theorem 15, more precisely Theorem 17 in the next section, the space $X$ can be constructed as follows.

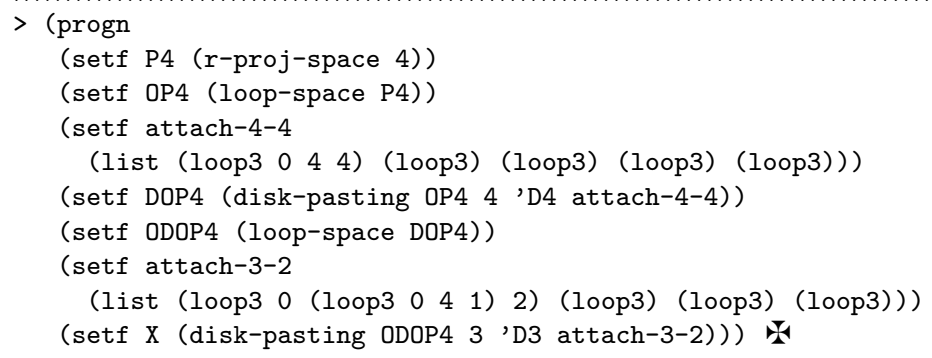

We cannot explain the technical details of the construction, but most of the statements are self-explanatory. Each object is located by a symbol and the assignment is set through a setf Lisp statement. For example the initial stunted projective space is assigned to the symbol P4. An object such as attach-4-4 describes an attaching map as a simplicial map $\partial \Delta^{4} \rightarrow$ OP4 and this description is then used by the Lisp function disk-pasting which constructs the desired space by attaching a cell according to the descriptor attach-4-4. The same for the end of the construction.

When this statement is executed, Lisp returns:

. .

(setf X (disk-pasting ODOP4 3 'D3 attach-3-2))) [K17 Simplicial-Set]

A maltese cross means the Lisp statement is complete, the read stage of the read-eval-print Lisp cycle is finished, the eval stage starts for an execution of the just read Lisp statement, it is the stage where the machine actually works, evaluating 
the statement. Most often, an object is returned (printed), it is the result of the evaluation process, in this case the simplicial set \#K17, located through the $\mathrm{X}$ symbol. This object $\mathrm{x}$ is a (machine) version with effective homology of the topological space $X$.

So we can ask for the effective homology of $X$; it is reached by the function efhm (effective homology) and assigned to the symbol SCE (strong chain equivalence):

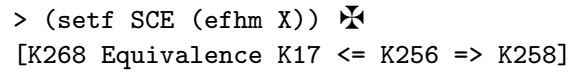

The Kenzo program returns a (strong) equivalence (Definition 13) between the chain complexes \#K17 and \#K258. Usually it is understood that a simplicial set produces an associated chain complex, but we may conversely consider that a simplicial set is nothing but a chain complex where a simplicial structure is added, compatible with the differential; for us, this is the right point of view and Kenzo follows this idea. Please compare with the discussion after Problem 2: a simplicial set is itself a chain complex with a further algebraic (!) structure. In other words, if you are only looking for an algebraic model for a homotopy type, the notion of simplicial set is a simple definitive solution, already given fifty years ago by Eilenberg and MacLane $[\mathbf{9 , 1 0}]$; this is the reason why a computability requirement is necessary to state a relevant problem.

In our equivalence describing the effective homology of $X$, the right chain complex \#K258 is effective, the left one \#K17 is not:

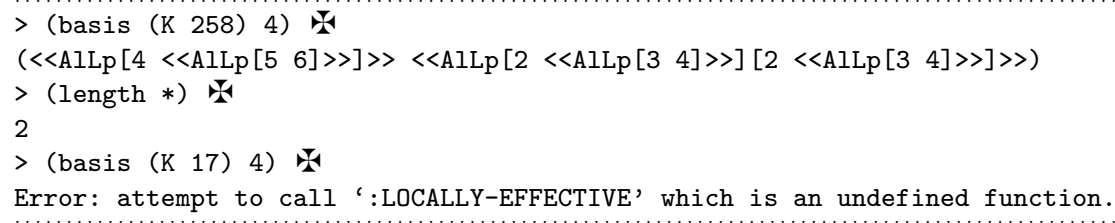

The basis in dimension 4 of the chain complex \#K258 is computed; it is a list of length $2\left({ }^{\prime} *\right.$ ' $=$ the last returned object). The elements of the basis themselves are "algebraic loops" (AlLp), elements of some appropriate cobar constructions.

You see it is not possible to obtain the basis in dimension 4 of the chain complex $\# \mathrm{~K} 17=C_{*} X$; the necessary functional object is in fact the keyword :locally-effective which generates an error.

A homology group of $X$ can be computed:

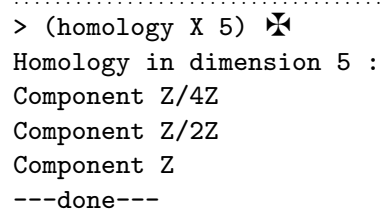

which means $H_{5} X=\mathbb{Z}_{4} \oplus \mathbb{Z}_{2} \oplus \mathbb{Z}$. It is in fact the homology of \#K258 in dimension 5 . 


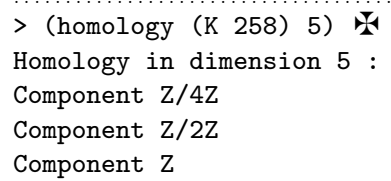

The strong chain equivalence \#K268 contains three chain complexes and two reductions, therefore four chain complex morphisms and two homotopy operators. In particular there is in the right reduction $\rho_{r}: K_{256} \ggg K_{258}$ a right $g: K_{258} \rightarrow K_{256}$ reachable by means of a $\underline{r g}$ function in the program; in the same way the left reduction $\rho_{\ell}: K_{17} K_{256}$ contains a left $f: K_{256} \rightarrow K_{17}$ reachable thanks to a If function. The Kenzo program can use these maps for arbitrary generators or combinations. For example the next Lisp statements play to verify the composite of the left $f$ and the right $g$ is compatible with the differentials.

We assign to the symbol gen the first generator of \#K258 in dimension 4, we apply the right $g(\mathrm{rg})$ to this generator, then the left $f(\mathrm{lf})$, finally the differential of \#K17:

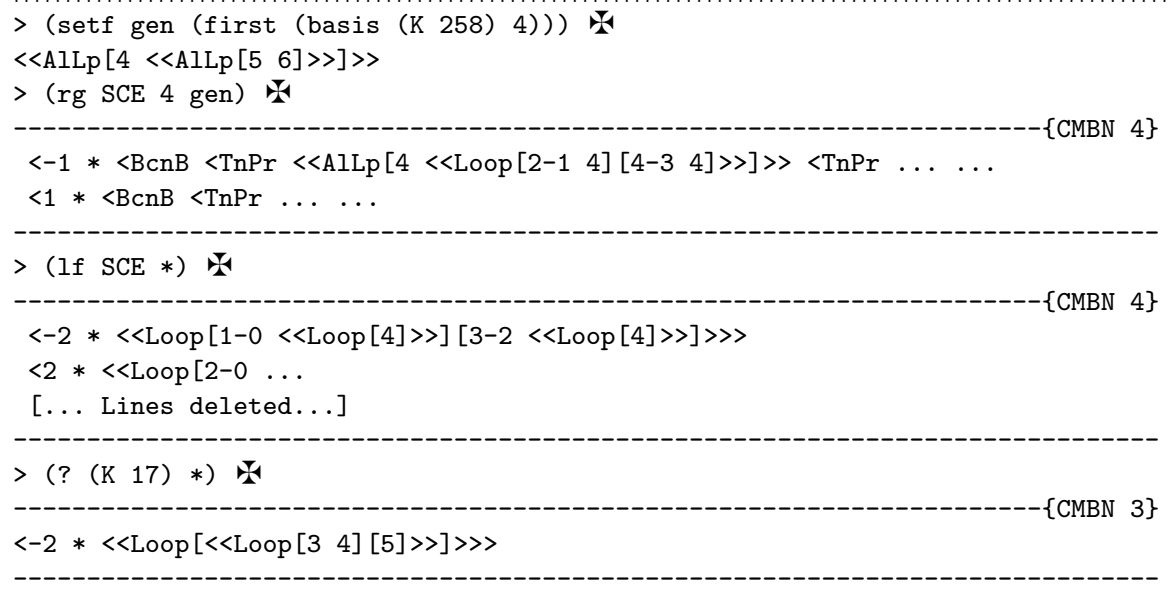

The result is a combination of degree 3 with a unique monomial, the coefficient is -2 and the generator is a "loop of loops", more precisely a simplex in the Kan model of $\Omega^{2}\left(P^{\infty} \mathbb{R} / P^{3} \mathbb{R}\right) \subset X$. Large parts of the intermediate results are not shown. A result between two dash lines '---' labeled for example \{CMBN 3$\}$ is a combination of degree 3 of integer coefficients and generators, one term per line.

The other path consists in applying to the same generator first the differential of \#K258 and then the same maps: 


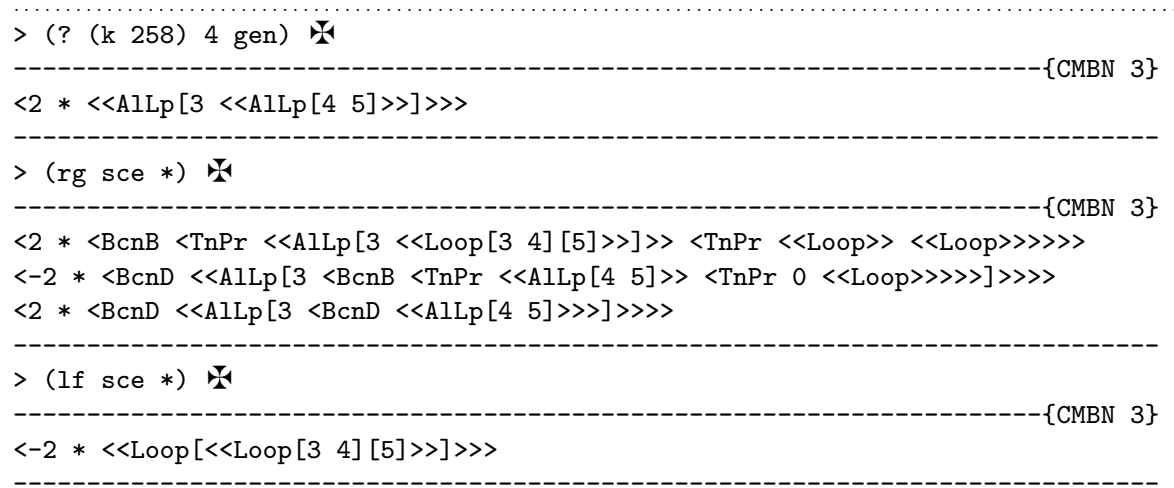

The results are the same. We will have more to say in Section 7.

\section{The fundamental theorem of Effective Homology.}

The classical spectral sequences (Serre, Eilenberg-Moore, Adams, ... ) are not algorithms. See for example [17, Section 1.1], in particular the comments following the unique theorem of the quoted section: most often, the available input for a spectral sequence does not determine the higher differentials. Something more is necessary for this essential problem, and it happens the category $\mathcal{S S}_{E H}$ is from this point of view a perfect solution; moreover it is a simple solution, once the possibilities of functional programming are understood.

Meta-Theorem 16. - Let

$$
\chi:\left(X_{i}\right)_{1 \leqslant i \leqslant n} \mapsto Y
$$

be a "reasonable" construction of the Algebraic Topology world producing $Y$ from the $X_{i}$ 's. Then an algorithm $\chi_{E H}$ can be written down which is a version with effective homology of the construction $\chi$ :

$$
\chi_{E H}:\left(\left(X_{i}\right)_{E H}\right)_{1 \leqslant i \leqslant n} \mapsto Y_{E H}
$$

Most often the $X_{i}$ 's and $Y$ are topological spaces. A construction is "reasonable" if it leads to some classical spectral sequence giving topologists the feeling that if the homology (for example) of the $X_{i}$ 's is known, then the homology of $Y$ might be "deduced".

A typical and important situation of this sort is the case where $X$ is a simply connected space and $\chi=\Omega$ is the loop space functor: $Y=\Omega X$. The EilenbergMoore spectral sequence gives interesting relations between $H_{*} X$ and $H_{*} \Omega X$, but this spectral sequence is not an algorithm computing $H_{*} \Omega X$ from $H_{*} X$, for a simple reason: it is possible that $H_{*} X=H_{*} X^{\prime}$ and $H_{*} \Omega X \neq H_{*} \Omega X^{\prime}$. More precisely, the cobar construction [1] gives the homology of the first loop space when some coproduct is available on $H_{*} X$, but the cobar construction does not give a coproduct on $H_{*} \Omega X$, so that the process cannot be iterated; this is Adams' problem: how to iterate the cobar construction? More than twenty years after Adams, Baues 
succeeded in a beautiful work [3] in iterating one time the cobar construction, giving the homology of the second loop space $\Omega^{2} X$ in reasonable situations, but Baues' method cannot be extended for the homology of $\Omega^{3} X$.

The category $\mathcal{S S}_{E H}$ gives at once a complete and simple solution for Adams' problem; it is a consequence of the following particular case of Meta-Theorem 16.

Theorem 17. - An algorithm $\Omega_{E H}$ can be written down:

$$
\Omega_{E H}: X_{E H} \mapsto(\Omega X)_{E H}
$$

producing a version with effective homology of the loop space $\Omega X$ when a version with effective homology of the initial simply connected space $X$ is given.

The algorithm $\Omega_{E H}$ not only can be written down, but it is written down; the algorithm $\Omega_{E H}$ is certainly the most important component of the Kenzo program [8], a program which currently is the most detailed description of the nature of Theorem 17 , a description not very convenient for an ordinary reader ${ }^{7}$.

The data type of the output $(\Omega X)_{E H}$ is exactly the same as the data type of the input $X_{E H}$, so that the algorithm $\Omega_{E H}$ can be trivially iterated.

Theorem 18. (Solution of Adams' problem ${ }^{8}$ ) - An algorithm ICB (iterated cobar) can be written down:

$$
\text { ICB : }\left(X_{E H}, n\right) \mapsto\left(\Omega^{n} X\right)_{E H}
$$

which produces a version with effective homology of the $n$-th loop space $\Omega^{n} X$ when a version with effective homology of the initial space $X$, assumed to be $n$-connected, is given.

When $X_{E H}=\left(X, C_{*} X, E C_{*}^{X}, \varepsilon^{X}\right)$ is given, the algorithm ICB produces a 4tuple $\left(\Omega^{n} X\right)_{E H}=\left(\Omega^{n} X, C_{*} \Omega^{n} X, E C_{*}^{\Omega^{n} X}, \varepsilon^{\Omega^{n} X}\right)$, where the " $n$-th cobar" of $E C_{*}^{X}$ is the third component $E C_{*}^{\Omega^{n} X}$. This $n$-th cobar cannot be constructed from $E C_{*}^{X}$ only; the first cobar needs the coproduct of $C_{*} X$ and the $n$-th cobar needs much more supplementary informations hidden in $X$ and $\varepsilon^{X}$ as indicated by V. Smirnov, M. Mandell and J. Smith ${ }^{9}$; these objects $X$ and $\varepsilon^{X}$ are locally effective and model mathematical objects which are infinite; yet $X$ and $\varepsilon^{X}$ are finite machine objects (pleonasm), namely finite bit strings actually created, processed and used by the Kenzo program; this process works thanks to functional programming.

The further components $\Omega^{n} X$ and $\varepsilon^{\Omega^{n} X}$ in the result would allow us to undertake other calculations starting from $\Omega^{n} X$.

\section{A small machine demonstration [sequel].}

Let us consider again the space $X$ of Section 5. The Kenzo program had constructed a version with effective homology of this space, allowing in particular the computation of its homology groups. Much more important, because of Theorem 17,

${ }^{7}$ See $[\mathbf{2 2}]$ for a survey which gives the plan and the main ideas of the proof of Theorem 17.

${ }^{8}$ This theorem is also a solution for Carlsson's and Milgram's problem [6, p. 545, Section 6].

${ }^{9}$ See also $[\mathbf{2}]$ for an analogous problem with respect to Lie algebras structures. 
the machine object $\Omega_{E H}$ of the same program can be applied to produce a version with effective homology of the loop space $\Omega X$ :

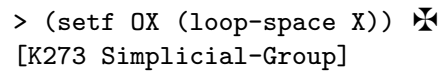

Kenzo $^{10}$ returns a new locally effective simplicial group, the Kan model of $\Omega X$ and its effective homology:

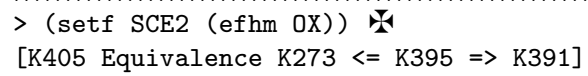

with which exactly the same experiences which were tried with the effective homology of $X$ in Section 5 could be repeated. In particular the right chain complex \#K391 is effective and allows a user to compute a homology group:

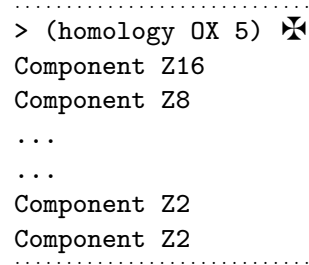

where we have deleted 21 lines, for the result is in fact:

$$
H_{5}\left(\Omega\left(\Omega\left(\Omega\left(P^{\infty}(\mathbb{R}) / P^{3}(\mathbb{R})\right) \cup_{4} D^{4}\right) \cup_{2} D^{3}\right)\right)=\mathbb{Z}_{16} \oplus \mathbb{Z}_{8} \oplus \mathbb{Z}_{2}^{23} .
$$

This is the corresponding homology group of \#K391.

To our knowledge, the Kenzo program is the only object, human or not, currently able to reach this result. See also $[\mathbf{2 0 , 2 6}]$ for two other interesting theoretical solutions which unfortunately have not yet led to concrete machine programs.

\section{The category $S S E H$ and the Postnikov category.}

A rough "definition" of the Postnikov category was given in Section 3.1, but we must now be more precise to obtain a correct relation between the category $\mathcal{S S}_{E H}$ and the Postnikov category.

Definition 19. (non-standard) - An Abelian group of finite type $\pi$ is a direct sum $\pi=\mathbb{Z} / d_{1} \mathbb{Z} \oplus \cdots \oplus \mathbb{Z} / d_{n} \mathbb{Z}$ where every $d_{i}$ is a non-negative integer and $d_{i-1}$ divides $d_{i}$. We denote by $\Pi$ the set of these groups.

The set $\Pi$ is designed for having exactly one group isomorphic to every Abelian group of finite type. For example the group $H_{5} X$ in Section 5 is isomorphic to the element of $\Pi$ defined by the integer triple $(2,4,0)$, that is, the group $\mathbb{Z}_{2} \oplus \mathbb{Z}_{4} \oplus \mathbb{Z}$, but there are 128 different isomorphisms.

${ }^{10}$ The Kenzo function loop-space follows the modern rules of Object Oriented Programming (OOP): if the argument is a simplicial set, then the Kan model of the loop space is constructed, and if furthermore the argument contains the effective homology of the initial simplicial set, then the loop-space function constructs also the effective homology of the loop space. 
Definition 20. - A Postnikov tower is a pair of sequences $\left(\left(\pi_{n}\right)_{n \geqslant 2},\left(k_{n}\right)_{n \geqslant 3}\right)$ where $\pi_{n} \in \Pi$ and $k_{n} \in H^{n+1}\left(X_{n-1}, \pi_{n}\right)$, where $X_{n}$ is the $n$-th stage of the Postnikov tower constructed by the standard process.

Because $\pi_{n}$ is some precise group, the standard Eilenberg-MacLane process gives a precise $K\left(\pi_{n}, n\right)$, a Kan simplicial set, producing in turn a precise $n$-th Postnikov stage $X_{n}$ and with the next $\pi_{n+1}$ a precise cohomology group $H^{n+2}\left(X_{n}, \pi_{n+1}\right)$ where the $k_{n+1}$ must be "chosen". A Postnikov tower so produces in a deterministic way a realization $X$. A morphism $f:\left(\pi_{n}, k_{n}\right) \rightarrow\left(\pi_{n}^{\prime}, k_{n}^{\prime}\right)$ between two Postnikov towers is a collection $\left(f_{n}: \pi_{n} \rightarrow \pi_{n}^{\prime}\right)$ of group homomorphisms compatible with the $k_{n}$ 's and $k_{n}^{\prime}$ 's, that is, satisfying Baues' relation, and we have so defined the Postnikov category $\mathcal{P}$. The isomorphism problem consists in deciding whether two Postnikov towers $\left(\pi_{n}, k_{n}\right)$ and $\left(\pi_{n}^{\prime}, k_{n}^{\prime}\right)$ produce realizations with the same homotopy type, that is, because of the context, that are isomorphic. Of course the condition $\pi_{n}=\pi_{n}^{\prime}$ is required for every $n$, but simple examples show that the condition $k_{n}=k_{n}^{\prime}$ on the contrary is not necessarily required. This is the reason why the $k_{n}$ 's are not invariants of the homotopy type.

The computable category $\mathcal{S S}_{E H}$ makes the realization process computable.

Theorem 21. - An algorithm PR (Postnikov realization) can be written down:

$$
\mathbf{P R}: \mathcal{P} \rightarrow \mathcal{S S}_{E H}
$$

implementing the realization process.

In fact the situation is significantly more complex. Before being. . true, the statement of this theorem must make sense, so that a machine implementation of the category $\mathcal{P}$ must be available, at least from a theoretical point of view. This is obtained thanks to the category $\mathcal{S S}_{E H}$ itself: a component $k_{n}$ must be a machine object, so that the data type $H^{n+1}\left(X_{n-1}, \pi_{n}\right)$ where $k_{n}$ is to be picked up must be previously defined, which is possible only if a calculation of this cohomology group can be undertaken. And again it is the category $\mathcal{S S}_{E H}$ which gives this possibility. It is an amusing situation where a category, the category $\mathcal{S S}_{E H}$, is simultanously used to give sense to the statement of a theorem, and synchronously finally to prove it.

Combining Theorem 21 with the appropriate particular cases of Meta-Theorem 16, we see the problem implicitly stated in the framed title inscription is now solved. In particular, the Kenzo program allows a Postnikov user to undertake many computations of this sort.

It is not possible, with the currently available tools, to make the categories $\mathcal{P}$ and $\mathcal{S S}_{E H}$ effectively equivalent, of course up to the homotopy relation.

Theorem 22. - An algorithm $\mathbf{S P}$ can be written down:

$$
\text { SP : } \quad \mathcal{S S}_{E H} \rightarrow \mathcal{P} \times \mathcal{I} \quad: \quad X \mapsto\left(\pi_{n}, k_{n}, I_{n}\right)_{n \geqslant 2}
$$

where the component $I_{n}$ is some isomorphism $I_{n}: \pi_{n}(X) \cong \pi_{n} \in \Pi=$ the set of "canonical" models of Abelian groups of finite type; the $k$-invariants $k_{n}$ are unambiguously defined only when the $I_{n}$ 's are chosen. 
The algorithm $\mathbf{S P}$ is essentially non-unique, for the choice of the component $I_{n}$ is arbitrary, and the various choices of these isomorphisms will produce all the possible collections of $k$ - "invariants" (!). Unfortunately the group $G L(p, \mathbb{Z})$ for example is infinite for $p>1$, so that it is a non-trivial arithmetical problem to determine whether two collections of $k$-invariants correspond to the same homotopy type or not. For example the problem has an obvious solution up to arbitrary dimensions if every $\pi_{n}$ is finite, but as soon as a $\pi_{n}$ is not finite, we are in front of interesting but difficult problems of arithmetic, to our knowledge not yet solved in general ${ }^{11}$.

In conclusion, thanks to the computable category $\mathcal{S S}_{E H}$, the category $\mathcal{P}$ becomes also a computable category. There are "good" but non-canonical correspondances between these categories. Both categories solve the soft problem and from this point of view give equivalent results. Both categories would solve the hard problem if the equivalence problem between systems of $k$ - "invariants" was effectively solved.

It is easier now to understand the common confusion about the nature of the $k$-invariants. We follow exactly here [16, §25], up to obvious slight differences of notations. If $X$ is a topological space, we can start with a minimal Kan model of $X$, "unique" up to numerous different isomorphisms in general; the Postnikov stages $X_{n-1}$ and $X_{n}$ are then canonical quotients of $X$. There is also a canonical fibration between $X_{n}$ and $X_{n-1}$, the fiber space of which being the canonical space $K\left(\pi_{n}(X), n\right)$, defining unambiguously a

$$
k_{n} \in H^{n+1}\left(X_{n-1}, \pi_{n} \underline{(X)}\right)=H^{n+1}\left(\underline{X} / \sim_{n-1}, \pi_{n} \underline{(X)}\right) .
$$

It is then clear that a claimed invariant living in $H^{n+1}\left(X_{n-1}, \pi_{n}\right)$ with $\pi_{n} \in \Pi$ depends on an isomorphism $\pi_{n} \cong \pi_{n}(X)$, which is essentially the $g$ correctly considered at [16, Theorem 25.7]. But if you think that $X_{n-1}$ comes only from the previous data $\pi_{2}, \pi_{3}, k_{3}, \ldots, \pi_{n-1}, k_{n-1}$ and not from $X$ itself, you can freely apply a self-equivalence of $X_{n-1}$ to change (!) the invariant, the fibration $X_{n} \rightarrow X_{n-1}$ is changed, but on the contrary the homotopy type of $X_{n}$ remains unchanged: different invariants correspond to equal homotopy types. The group of all the self-equivalences of $X_{n-1}$ must be considered, of course in general a serious question. One way to cancel this ambiguity consists in choosing a well defined partial equivalence between $X$ and $X_{n-1}$, which amounts to choosing some isomorphisms $\pi_{i} \cong \pi_{i}(X)$ for $2 \leqslant i<n$.

\footnotetext{
${ }^{11}$ Compare with [20, pp. 54-59]; the possible equivalence of $k_{n}$ and $k_{n}^{\prime}$ with respect to some automorphism of the last $\pi_{n}$ is there proved decidable, which is relatively easy. But this does not seem to be sufficient, because the possible automorphisms of all the previous $\pi_{m}, m \leqslant n$, must be considered. The example of a Postnikov tower where only $\pi_{2}=\mathbb{Z}^{p}$ and $\pi_{5}=\mathbb{Z}$ given in Section 3.1 shows the main problem for the equivalence of $k$-invariants is in the automorphisms of $\pi_{2}$, because the group of automorphisms is $G L(p, \mathbb{Z})$, leading to an arithmetical problem which seems open. In a later preprint, not published, Rolf Schön considers again the problem, solves it in the case where all the $\pi_{n}$ 's are finite, and announces a general solution which "takes considerable work". The authors have not succeeded in getting in touch with Rolf Schön for several years, and any indication about his current location would be welcome. Note that these comments in particular cancel the assertion in [23, Section 5.4] about the classification problem, which assumed the correctness of Schön's paper: the solutions called JS, SRH and SRG in [23] solve only the soft problem; an essential gap remains present for the hard problem.
} 
Maybe it is useful to recall an invariant with respect to any notion must be chosen in a "fixed" world independent of the object the invariant of which is being defined. Otherwise the definitively simplest complete invariant for the homotopy type of $X$ is $X$ itself. Not very interesting. In [16, Theorem 25.7] the definition $k_{n} \in H^{n+1}\left(X_{n-1}, \pi_{n}\right)$ (p. 113, line 19) and the notation $\pi_{n}=\pi_{n}(X, \emptyset)$ (line 14) contain two illegal occurrences of $X$ when defining an invariant: an elementary rule of formal logic is not satisfied. It is the reason why, in the statement of Theorem 22, $\pi_{n}$ is not equal to $\pi_{n}(X)$, they are only isomorphic through some isomorphism which plays an essential role.

The classical example of the minimal polynomial of a matrix is helpful; if the ground field $K$ is given, then the minimal polynomial can be chosen once and for all in $K[\lambda]$, a set of polynomials independent of the particular considered matrix. So that if two matrices are conjugate, more generally if two endomorphisms of two finite-dimensional $K$-vector spaces are conjugate, their minimal polynomials are equal, not mysteriously "isomorphic"; this is the reason why the miminal polynomial is a correct conjugation invariant.

\subsection{Localization.}

Another natural idea must also be considered. It is usual to split a topological problem $P$ into a rational problem $P_{0}$ and $p$-problems $\left(P_{p}\right)_{p \in \mathfrak{P}}$ for $p$ running the prime numbers $\mathfrak{P}$. Then a solution for every problem $P_{p}$ including $p=0$ theoretically produces a solution for the initial problem $P$. Let us take again our fetish example of the Postnikov towers with only $\pi_{2}=\mathbb{Z}^{k}$ and $\pi_{2 n-1}=\mathbb{Z}$ where the $k_{2 n-1}$-invariant is $\chi \in S_{k}^{n}$, the $\mathbb{Z}$-module of the homogeneous $\mathbb{Z}$-polynomials of degree $n$ with respect to $k$ variables. The localization method produces a localized Postnikov tower $T_{p}$ for every element $p \in\{0\} \cup \mathfrak{P}$. We are then in front of a list of problems.

Problem 23. - Let $\chi$ and $\chi^{\prime}$ be two polynomials defining Postnikov towers $T$ and $T^{\prime}$, producing in turn families of Postnikov towers $\left(T_{p}\right)$ and $\left(T_{p}^{\prime}\right)$.

1. Let $p$ be an element of $\{0\} \cup \mathfrak{P}$; can the mapping $T \mapsto T_{p}$ be made effective?

2. Can the isomorphism problem between $T_{p}$ and $T_{p}^{\prime}$ be effectively solved?

3. Does there exist an integer $p_{0}$ and an argument allowing us to be exempt from this study for $p>p_{0}$ ?

4. If the isomorphism problem between $T_{p}$ and $T_{p}^{\prime}$ has a positive effective solution for every $p$, does there exist an effective process allowing the construction of an isomorphism between $T$ and $T^{\prime}$ ?

Subproblems 1 and 2 of Problem 23 probably are "exercises", but the subproblems 3 and 4 seem serious. Notice again a general solution for Problem 23 would solve at once the problem of the $\mathbb{Z}$-linear equivalence between elements of $S_{k}^{n}$ for every $n$, while the arithmeticians currently know the solution only for $n=2$, a non-trivial problem [7]. From this point of view, it would be interesting to translate the known solution which is available for $n=2$ into a solution of Problem 23 . 


\section{The category $\mathcal{S S}_{E H}$ and the $E_{\infty}$-algebras.}

We had briefly mentioned in Section 3.2 the possibility of other solutions for the hard problem based on $E_{\infty}$-operads. Exactly as in the previous section, the $p$-localized versions [13] of these operads can be considered, or if it is preferred directly the $\mathbb{Z}$-version $[\mathbf{1 4}]$. These versions lead to a correspondance problem analogous to Problem 23. The resulting classification problem in this framework consists in determining whether some quasi-isomorphism of $E_{\infty}$-chain complexes can be installed between two such given chain complexes. Taking account of the numerous tensor products involved in the usual descriptions of $E_{\infty}$-operads, we see we are again in front of some sophisticated linear equivalence problems between generalized polynomials: the nature of our hard problem, when expressed in this setting, seems exactly the same.

A particularly interesting $E_{\infty}$-operad is the surjection operad $\mathcal{S}$ defined and studied in [5], a work undertaken to make completely explicit some results ${ }^{12}$ of Mandell's paper [14] already quoted in Section 3.2. The so-called surjection operad and its action on a simplicial set can be understood as a "complete" generalization of the Steenrod operations, and we therefore propose to call it the Steenrod operad, which furthermore allows us to keep the same notation $\mathcal{S}$.

Theorem 24. - A functorial algorithm SSC (simplicial sets to Steenrod chain complexes) can be written down:

$$
\text { SSC : } \mathcal{S S}_{E H} \rightarrow \mathcal{C C}_{\mathcal{S}}
$$

where $\mathcal{C C}_{\mathcal{S}}$ is the category of the free $\mathbb{Z}$-chain complexes of finite type provided with a $C B \mathcal{S}$-operadic structure.

An appropriate bar construction can be applied to the operad $\mathcal{S}$ to produce a cooperad $B \mathcal{S}$; then an analogous cobar construction can in turn be applied to this cooperad to produce a new operad denoted by $C B \mathcal{S}$, another model for an $E_{\infty^{-}}$ operad which has the following advantage ${ }^{13}$ : let $f: C_{*} \rightarrow D_{*}$ be a chain equivalence between two free $\mathbb{Z}$-chain complexes; then every $C B \mathcal{S}$-structure on $C_{*}$ induces such a structure on $D_{*}$.

Definition 25. - A Steenrod chain complex is a free $\mathbb{Z}$-chain complex provided with an $\mathcal{S}$-structure or with a $C B \mathcal{S}$-structure.

Let $X$ be an object of $\mathcal{S} \mathcal{S}_{E H}$, that is, a simplicial set with effective homology. The article [5] explains how the initial definition by Steenrod of his famous cohomological operations can be naturally used to install a canonical $\mathcal{S}$-structure on the chain complex $C_{*} X$; the strong chain equivalence $\varepsilon^{X}: C_{*} X C_{*}^{X}$ then allows us to install a $C B \mathcal{S}$-structure on $E C_{*}^{X}$, and this is enough to define the functor SSC.

Taking account of Mandell's article [14], the following problems are natural.

${ }^{12}$ The considerations of Section 8.1 can again be applied when comparing the surjection operad $\mathcal{S}$, a $\mathbb{Z}$-operad, and the "abstract" $p$-localized operads of Mandell.

${ }^{13}$ We would like to thank Tornike Kadeishvili for his clear and useful explanations about this process. 
Problem 26. - Does there exist an algorithm $\mathbf{R}$ (realizability):

$$
\mathbf{R}: \mathcal{C C}_{\mathcal{S}} \times \mathbb{N} \rightarrow \text { Bool }=\{\top, \perp\}
$$

allowing us to decide whether some object $C_{*} \in \mathcal{C C}_{\mathcal{S}}$ corresponds or not to some topological object up to some given dimension?

Because of the Characterization Theorem [13, p. 2], a solution for this problem is probably a "simple" exercise, simple in theory but the operad $C B \mathcal{S}$ is rather sophisticated, so that a concrete solution seems a nice challenge. Furthermore the Characterization Theorem is stated and proved in characteristic $p$ and obtaining the analogous result with respect to the ground ring $\mathbb{Z}$ could be a little difficult.

Problem 27. - Does there exist an algorithm SHT (same homotopy type):

$$
\text { SHT : } \mathcal{C C}_{\mathcal{S}}^{\prime} \times \mathcal{C C}_{\mathcal{S}}^{\prime} \times \mathbb{N} \rightarrow \text { Bool }
$$

allowing us to decide whether two $\mathcal{C C}_{\mathcal{S}}$-objects obtained through the $\mathbf{S S C}$-algorithms, therefore certainly corresponding to actual recursive simplicial sets, have the same homotopy type or not, of course up to some given dimension?

The authors are not sufficiently experienced in operadic techniques to estimate the difficulty of this question. The Main Theorem of [13, p. 1] seems to imply that the same considerations as for Problem 26 could be applied; but as already observed, an effective solution of Problem 27 would indirectly solve crucial computability problems in arithmetic, problems which seem to raise essential obstacles confronting the professionals.

If the operadic methods become unavoidable, it seems terribly difficult to design directly the category $\mathcal{C C}_{\mathcal{S}}$ as a computable category. We think it would be more sensible to work simultaneously with the categories $\mathcal{S S}_{E H}$ and $\mathcal{C C}_{\mathcal{S}}$ : it is frequent in mathematics in general, and in computer science in particular, that it is not a good idea to give up too early information which looks redundant. This is well known for example by the theoreticians in homotopy theory: it is much better to work with an explicit homotopy equivalence than only with the existence of such an object, and it is still better to keep also the various maps which describe how this homotopy equivalence actually is one, and so on. This is nothing but the philosophy always underlying work with $E_{\infty}$-operads.

In our situation, Theorem 24 implies a simplicial set with effective homology $X_{E H}$ contains in an effective way a Steenrod chain complex; and we do not need any realizability criterion, an object of $\mathcal{S S}_{E H}$ certainly corrresponds to a genuine topological space. Therefore good objects to work with in Algebraic Topology could be the pairs $\left(X_{E H}, \Sigma_{\mathcal{S}}^{X}\right)$ where the second component $\Sigma_{\mathcal{S}}^{X}$ is the $C B \mathcal{S}$-structure induced on $E C_{*}^{X}$ by the canonical Steenrod structure on $C_{*} X$. Then, when a new object is constructed from such objects, the ingredients present in the second components could facilitate the computation of some parts of the constructed object, but others would certainly be obtained much more easily, thanks to the first components.

In a sense the success of the category $\mathcal{S S}_{E H}$ is already of this sort: instead of working only with a chain complex $E C_{*}^{X}$ describing the homology of $X$, certainly 
in general not sufficient for the planned computations, it is much better to work with $X$ itself under its locally effective form, the only form which can be processed on a machine when $X$ is not of finite type. The amazing fact is that this is sufficient to solve many computability problems, though this version of $X$ does not effectively define the mathematical object $X$, because of Gödel and his friends, see [23, Section 5.3]. The same people, helped by Matiyasevich [15], have also made impossible a universal solver of systems of polynomial $\mathbb{Z}$-equations, and after all, the hard problem is equivalent to a problem about such equations, so that we cannot even be sure, up to further information, a solution of the hard problem exists.

\section{References}

[1] J. F. Adams, Peter J. Hilton. On the chain algebra of a loop space. Commentarii Mathematici Helvetici, 1956, vol. 30, pp 305-330.

[2] Samer Al-Ashab. A class of strongly homotopy Lie algebras with simplified sh-Lie structures. arXiv:math.RA/0308160.

[3] Hans J. Baues. Geometry of loop spaces and the cobar construction. Memoirs of the American Mathematical Society, 1980, vol. 230.

[4] Hans J. Baues. Homotopy types. in [12], pp 1-72.

[5] Clemens Berger and Benoit Fresse. Combinatorial operad actions on cochains. To appear in Math. Proc. Cambridge Philos. Soc.

www-gat.univ-lille1.fr/ fresse/CochainModel.pdf

[6] Gunnar Carlsson and R. James Milgram. Stable homotopy and iterated loop spaces. In [12], pp 505-583.

[7] J. W. S. Cassels. Rational quadratic forms. Academic Press, 1978.

[8] Xavier Dousson, Julio Rubio, Francis Sergeraert and Yvon Siret. The Kenzo program. http://www-fourier.ujf-grenoble.fr/ sergerar/Kenzo/

[9] Samuel Eilenberg, Saunders MacLane. On the groups $H(\pi, n)$, I. Annals of Mathematics, 1953, vol. 58, pp 55-106.

[10] Samuel Eilenberg, Saunders MacLane. On the groups $H(\pi, n)$, II. Annals of Mathematics, 1954, vol. 60, pp 49-139.

[11] Encyclopedic Dictionary of Mathematics, sub-article Postnikov complexes, in different articles according to the edition (look for Postnikov complex in the final index). Mathematical Society of Japan and American Mathematical Society.

[12] Handbook of Algebraic Topology (Edited by I.M. James). North-Holland (1995).

[13] M. Mandell. $E_{\infty}$-algebras and p-adic homotopy theory. Topology, 2001, vol. 40, pp 43-94.

[14] Michael A. Mandell. Cochains and homotopy types. arXiv:math.AT/0311016.

[15] Yuri Matiyasevich. Hilbert's tenth problem. MIT Press, 1993.

[16] J. Peter May. Simplicial objects in algebraic topology. Van Nostrand, 1967. 
[17] John McCleary. User's guide to spectral sequences. Publish or Perish, Wilmington DE, 1985.

[18] Julio Rubio, Francis Sergeraert. Constructive Algebraic Topology. Bulletin des Sciences Mathématiques, 2002, vol. 126, pp 389-412.

[19] David E. Rydeheard, Rod M. Burstall. Computational Category Theory. Prentice Hall, 1988.

[20] Rolf Schön. Effective algebraic topology. Memoirs of the American Mathematical Society, 1991, vol. 451.

[21] Francis Sergeraert. The computability problem in algebraic topology. Advances in Mathematics, 1994, vol. 104, pp 1-29.

[22] Francis Sergeraert. Effective homology, a survey. www-fourier.ujf-grenoble.fr/ sergerar/Papers/survey.dvi or ps.

[23] Francis Sergeraert. ${ }_{K}$, objet du $3^{e}$ type. Gazette des Mathématiciens, 2000, vol. 86, pp 29-45.

[24] Francis Sergeraert. www-fourier.ujf-grenoble.fr/ sergerar/Papers/.

[25] Vladimir A. Smirnov. The homology of iterated loop spaces. Forum Mathematicum, 2002, vol. 14, pp 345-381.

[26] Justin R. Smith. Iterating the cobar construction. Memoirs of the American Mathematical Society, 1994, vol. 524.

[27] A.S. Troelstra, D. van Dalen. Constructivism in mathematics, an introduction. North-Holland, 1988.

This article may be accessed via WWW at http://www.rmi.acnet.ge/hha/ or by anonymous ftp at

ftp://ftp.rmi.acnet.ge/pub/hha/volumes/2005/n2a8/v7n2a8.(dvi,ps,pdf)

Julio Rubio Julio.Rubio@dmc.unirioja.es

Departamento de Matemáticas y Computación

Universidad de La Rioja

Edificio Vives. Calle Luis Ulloa s/n

26004 Logroño, La Rioja

Spain

Francis Sergeraert Francis.Sergeraert@ujf-grenoble.fr

Institut Fourier

Université Joseph Fourier

BP 74

38402 St Martin d'Hères Cedex

France 